\title{
A GREEN'S THEOREM IN TERMS OF LEBESGUE INTEGRALS.
}

Br H. E. Brat.

The present paper contains the proof of the Green's Theorem which is associated with the integral form of Poisson's equation, that is to say, with the equation:

$$
\int_{c} \frac{\partial u}{\partial x} d y-\frac{\partial u}{\partial y} d x=\iint_{R} f(x, y) d x d y
$$

The method of proof, that of approximating polynomials, was suggested by Professor G. C. Evans, who uses it in the proof of a similar theorem,* where, however, the functions $u, \partial u / \partial x, f$, etc., have to obey certain restrictions as to continuity owing to the fact that the integrals in the equation are of the ordinary kind. Here, however, Lebesgue integrals are used; consequently, as might be expected, the properties of $u, \partial u / \partial x$, $f$, etc., are less restricted.

The region, $R$, here considered is a rectangle.

The works cited in footnotes are the Cours d'analyse of de la ValléePoussin and the article Sur l'integrale de Lebesgue by the same author in the Transactions of the American Mathematical Society, Volume XVI, 1916. They are referred to, briefly, as Cours d'analyse and Transactions respectively.

$\S 1$. The following theorems and definitions will be used in the course of this discussion.

Theorem $A . \dagger$ If the transformation

$$
x_{1}=\varphi\left(y_{1}, y_{2}\right), \quad x_{2}=\psi\left(y_{1}, y_{2}\right)
$$

establishes a one-to-one continuous correspondence between two measurable sets, $E_{x}$ and $E_{y}$, and if, moreover, the formulæ of the transformation are differentiable on $E_{y}$, at every point of $E_{y}$; and if $f\left(x_{1}, x_{2}\right)$ is summable in $E_{x}$, then $F \cdot|J| \ddagger$ will be summable on $E_{y}$, and

$$
\int_{F x} f\left(x_{1}, x_{2}\right) d P_{x}=\int_{F y} f|J| d P_{y}
$$

provided that we agree to put $f \cdot|J|=0$ at every point where $|J|$ vanishes, even when $f$ becomes infinite.

* Cambridge Colloquium Lectures, September, 1916.

$\dagger$ De la Vallee Poussin, Transactions, p. 500.

$\ddagger J$ is the Jacobian of the transformation. 
The theorem can be extended to the case of more than two variables.

Throras $B$. If $u(x, y)$ is an absolutely continuous function of $y$ for every value of $x(a \leq x \leq b, c \leq y \leq d)$, and is summable linearly with regard to $x$, for every value of $y$, and if $\partial u / \partial y$ is summable superficially in the same region, then

$$
f(y)=\int_{a}^{b} u(x, y) d x
$$

is an absolutely continuous function of $y$.

For since $\partial u / \partial y$ is summable superficially, and $u$ linearly,

$f\left(y^{\prime \prime}\right)-f\left(y^{\prime}\right)=\int_{a}^{b}\left[u\left(x, y^{\prime \prime}\right)-u\left(x, y^{\prime}\right)\right] d x$

Therefore

$$
=\int_{a}^{b} d x \int_{\gamma^{\prime}}^{s^{\prime \prime}} \frac{\partial u}{\partial y} d y=\int_{R\left(a, b ; p_{p}\right)} \int \frac{\partial u}{\partial y} d x d y .
$$

$$
\sum_{i=1}^{\infty}\left[f\left(y_{i+1}\right)-f\left(y_{i}\right)\right]=\int_{\Sigma} \int \frac{\partial u}{\partial x} d x d y,
$$

where $E$ is a denunserable set of rectangles $R_{i}\left[y_{i} \leq y \leq y_{i+1}, a \leq x \leq b\right]$. Since the double integral is absolutely continuous, $\sum_{i=1}^{\infty}\left[f\left(y_{i+1}\right)-f\left(y_{i}\right)\right]$ approaches zero with $m(E)$, i.e., with $\sum_{i=1}^{\infty}\left(y_{i+1}-y_{i}\right)$. Thus our theorem is proved.

Oniform absolute continuity.* Consider a sequence of summable functions $f_{\mu}(x)$ which converge to the function $f(x)$ over the set $E$.

Deftnition. The absolute continuity of the integrals

$$
\int f_{\mu} d x
$$

is said to be uniform over the set $E$, if to every positive $\epsilon$ there corresponds a \& such that

$$
\left|\int_{\varepsilon} f_{n} d x\right|<\epsilon
$$

independent of $\mu$, provided that $e$ be a portion of $E$, of measure less than $\delta$ (Vitali).

Throrer $C . t$ If the absolute continuity of the integrals

$$
\int f_{k}(x) d x
$$

- de la Vallee Poussin, Transactions, pp. 445 et seq.

t de la Vallee Poursin, loc. cit.

$$
\begin{gathered}
\text { Thesis } \\
\text { Math. } \\
\text { laig } \\
\text { Bray } \\
\text { c.l }
\end{gathered}
$$


is uniform over the set $E, f(x)$ is summable over $E$ and

$$
\lim _{x=\infty} \int_{\Sigma} f_{n} d x=\int_{\Sigma} f(x) d x .
$$

The definition of absolute continuity and the proof of the theorem (C) can be extended to the case where the functions $f_{n}$ involve more than one variable.

We generalize the notion of uniform absolute continuity as follows:

Definmon. Let us suppose that the function $f(x, y, \alpha)$ is summable over the set $E(x, y)$ for all values of $\alpha$ belonging to a set $A(\alpha)$. Then if the integral

$$
F(\alpha)=\int_{\varepsilon} \int f(x, y, \alpha) d x d y
$$

is such that to every positive $\epsilon$ there corresponds a $\delta$ such that

$$
\left|\iint f(x, y, \alpha) d x d y\right|<\epsilon
$$

for all values of $\alpha$ in $A$, provided only that $m(e)<\delta$, the absolute continuity of the integral $F(\alpha)$ is said to be uniform with regard to $\alpha$.

This definition includes the previous one.

Thropra $D$. If $u(x, y)$ is limited and summable (linearly) with regard to $y$ for all values of $x$ in the region $[a \leq x \leq b, c \leq y \leq d]$ and if $u$ is absolutely continuous in $x$ for all values of $y$, and if $\partial u / \partial x$ is summsble superficially in the same region, then the function

$$
F(x, y)=\int_{a}^{v} u(\dot{x}, \eta) d \eta
$$

is a continuous function of the two variables $x$ and $y$. For consider the expression

$$
\begin{aligned}
F(x, y)-F\left(x_{0}, y_{0}\right) & \\
& =\int_{a}^{y} u(x, \eta) d \eta-\int_{a}^{v_{0}} u(x, \eta) d \eta+\int_{a}^{v_{0}} u(x, \eta) d \eta-\int_{a}^{v_{0}} u\left(x_{0}, \eta\right) d \eta \\
& =\int_{v_{0}}^{\eta_{0}} u(x, \eta) d \eta+\int_{a}^{m_{0}}\left[u(x, \eta)-u\left(x_{0}, \eta\right)\right] d \eta .
\end{aligned}
$$

The first term can be made as small as we please by taking $\left|y-y_{0}\right|$ small enough, independent of $x$, since $u$ is limited. The second term can be written

$$
\int_{a}^{y_{0}} d \eta \int_{x_{0}}^{x} \frac{\partial u}{\partial \xi} d \xi=\int_{R\left(x_{0,} \times a_{1}, \eta_{0}\right)} \int \frac{\partial u}{\partial \xi} d \xi d \eta
$$


since $u$ is absolutely continuous in $x$, and $\partial u / \partial \xi$ is summable superficially. For the latter reason the integral

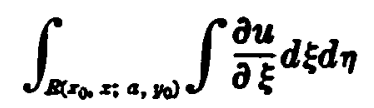

approaches zero with $m(R)$, i.e., with $\left|x-x_{0}\right|$. Hence finally, $F(x, y)$ $-F\left(x_{0}, y_{0}\right)$ approaches zero with $\sqrt{\left(x-x_{0}\right)^{2}+\left(y-y_{0}\right)^{2}}$, and this is what we wished to prove.

\$ 2. I. Theorex. If (1) $u(x, y), v(x, y)$, are limited and summable (superficially) throughout the region $R(0 \leq x \leq 1,0 \leq y \leq 1)$,

(2) $u$, likewise $v$, is an absolutely continuous function of $x$ for every value of $y$, and of $y$ for every value of $x$,

(3) $\partial u / \partial x, \partial u / \partial y, \partial v / \partial x, \partial v / \partial y$, considered where they exist, are summable (superficially) in $R$,

(4) $f(x, y), g(x, y)$ are summable in $R$,

(5) the following equations are satisfied for every rectangle $D\left[X_{1} \leq x \leq X_{2}\right.$, $Y_{1} \leq y \leq Y_{2}$ inside $R$, for which the left-hand members have a meaning:

$$
\begin{aligned}
& \int_{c} \frac{\partial u}{\partial x} d y-\frac{\partial u}{\partial y} d x=\iint_{D} f(x, y) d x d y \\
& \int_{c} \frac{\partial v}{\partial x} d y-\frac{\partial v}{\partial y} d x=\iint_{D} g(x, y) d x d y,
\end{aligned}
$$

(6) the rectangle $S[a \leq x \leq b, c \leq y \leq d]$ is such that the absolute continuity of the integral $\boldsymbol{S}(\partial u / \partial x) d y$ is uniform in the neighborhood of $x=a, x=b$; similarly the absolute continuity of $\mathcal{S}(\partial u / \partial y) d x$ is uniform in the neighborhood of $y=c, y=d$,

(7) at nearly every point of the boundary of $S, u, \partial u / \partial x, \partial u / \partial y, v$, $\partial v / \partial x, \partial v / \partial y$ are the superficial derivatives of their respective double integrals $\int \boldsymbol{S} u d x d y$, etc.

then for every such rectangle $S$

$$
\int_{c}\left(v \frac{\partial u}{\partial x}-u \frac{\partial v}{\partial x}\right) d y-\left(v \frac{\partial u}{\partial y}-u \frac{\partial v}{\partial y}\right) d x=\iint_{s}(v f-u g) d x d y \text {. }
$$

It is to be noted that, since $u, \partial u / \partial x, \partial u / \partial y$, etc., are summable superficially, the values of $a,(c)$, for which the lines $x=a,(y=c)$, do not satisfy condition (7), form a set of zero measure.* Also, for the same reason, the values of $X_{1}, X_{2}, Y_{1}, Y_{2}$, for which the left-hand members of equations $(A),(B)$ do not have a meaning, form a set of zero measure. $\dagger$ Hence conditions (5) and (7) are satisfied by nearly every rectangle in $\boldsymbol{R}$.

- A summable function is the derivative of its indefinite integral nearly everywhere.

† Cours d'analyse, vol. II, pp. 117 et seq. 
A GREEN'S THEOREM IN TERMS OF LEBESGUE INTEGRALS.

Similarly, since $u, v$ are limited, equation $(C)$ will have a meaning for nearly every rectangle $S$ in $R$.

II. The proof of the theorem is obtained by the use of approximating polynomials, * defined as follows:

$$
\bar{P}_{\mu}[u(x, y)]=\frac{1}{k_{\mu}^{2}} \iint_{R} u(\xi, \eta)\left[1-\left(\xi-x^{2}\right)\right]^{\mu}\left[1-(\eta-y)^{2}\right]^{\star} d \xi d \eta,
$$

where

$$
\begin{gathered}
k_{\mu}=2 \int_{0}^{l}\left(1-t^{2}\right)^{\mu} d t, \\
P_{\mu}[u(x, y)]=\frac{1}{k_{\mu}^{2}} \int_{-x}^{1-x} d \xi \int_{-y}^{1-y} u(\xi+x, \eta+y)\left[1-\xi^{2}\right]^{\mu}\left[1-\eta^{2}\right]^{\mu} d \eta .
\end{gathered}
$$

Let

$$
\bar{P}_{\mu}[u(x, y)]=\frac{1}{k_{\mu}^{2}} \int_{x=\varepsilon}^{x+e} d \xi \int_{y=\varepsilon}^{\nu+\varepsilon} u(\xi, \eta)\left[1-(\xi-x)^{2}\right]^{\mu}\left[1-(\eta-y)^{2}\right]^{\mu} d \eta,
$$

where $\epsilon$ is a positive number, less than any of the numbers $a, 1-b, c$, 1 - d. Evidently

$$
P_{\mu}[u(x, y)]=\frac{1}{k_{\mu}^{2}} \int_{-a}^{e} d \xi \int_{-\varepsilon}^{e} u(\xi+x, \eta+y)\left[1-\xi^{2}\right]^{\mu}\left[1-\eta^{2}\right]^{z} d \eta .
$$

It is well known that $P_{k}[u(x, y)]$ approaches $u(x, y)$ nearly everywhere in $S$, and therefore by condition ( 7 ) nearly everywhere on the boundary of $S$, as $\mu \doteq \infty$. It is to be noted that the functions $P_{\mu}[u(x, y)], \bar{P}_{\alpha}[u(x, y)]$ are limited in their sets. It is also well known that

$$
Q_{\mu}[u]=P_{\mu}[u]-\bar{P}_{\mu}[u]
$$

approaches zero and that $\bar{P}_{\mu}[u]$ approaches $u$ as $\mu \doteq \infty$.

III. The method of proof is as follows: Since $P_{\mu}[u], P_{\mu}[v]$ are polynomials, they satisfy the conditions of Green's Theorem, as usually stated, and we can therefore write:

$$
\begin{array}{r}
\int_{c}\left\{P_{\mu}[v] \frac{\partial P_{\mu}[u]}{\partial x}-P_{\mu}[u] \frac{\partial P_{\mu}[v]}{\partial x}\right\} d y-\left\{P_{\mu}[v] \frac{\partial P_{\mu}[u]}{\partial y}-P_{\mu}[u] \frac{\partial P_{\mu}[v]}{\partial y}\right\} d x \\
=\iint_{S}\left\{P_{\mu}[v] \nabla^{2} P_{\mu}[u]-P_{\mu}[u] \nabla^{2} P_{\mu}[v]\right\} d x d y .
\end{array}
$$

It is proved that, on the boundary of $S$

$$
\lim _{x=\infty} \frac{\partial P_{u}[u]}{\partial x}=\frac{\partial u}{\partial x}, \quad \lim _{\kappa=\infty} \frac{\partial P_{u}[u]}{\partial y}=\frac{\partial u}{\partial y}
$$

* Cours d'analyse, vol. II, pp. 126 et seq. 
and that, in $S$

$$
\lim _{u \in \infty} \nabla^{2} P_{\alpha}[u]=f(x, y)
$$

and similar relations are proved for $P_{k}[v]$. It is shown moreover that the limits of the above integrals, in (1), are equal to the integrals of the limits of their integrands. We thus obtain, by taking the limits of both sides of (1), the formula to be proved:

$$
\int_{\epsilon}\left(\frac{v u}{\partial x}-u \frac{\partial v}{\partial x}\right) d y-\left(v \frac{\partial u}{\partial y}-u \frac{\partial v}{\partial y}\right) d x=\iint(v f-u g) d x d y .
$$

We have to prove that the absolute continuity of the integrals in (1) is uniform with regard to $\mu$ (Theorem $C$ ).

IV. We consider now the quantity:

$$
Q_{\mu}[u(x, y)]=P_{\mu}[u(x, y)]-\bar{P}_{\mu}[u(x, y)] .
$$

A glance at the diagram of the regions of integration of $P_{\mu}[u]$ and $\bar{P}_{\alpha}[u]$ will show that $Q_{\mu}[u]$ is the sum of eight terms of which the following are typical:

$$
\begin{aligned}
& \alpha_{k}[x, y]=\frac{1}{k_{\mu}^{2}} \int_{y+\varepsilon}^{1} d \eta \int_{x+\varepsilon}^{l} u(\xi, \eta)\left[1-\left(\xi-x^{2}\right)\right]^{\kappa}\left[1-(\eta-y)^{2}\right]^{\mu} d \xi, \\
& \beta_{\mu}[x, y]=\frac{1}{k_{\mu}{ }^{2}} \int_{y-\varepsilon}^{y+\varepsilon} d \eta \int_{x+e}^{1} u(\xi, \eta)\left[1-(\xi-x)^{2}\right]^{\mu}\left[1-(\eta-y)^{2}\right]^{\mu} d \xi \text {. }
\end{aligned}
$$

With regard to $\alpha_{n}$, we see that the inner integral is an absolutely continuous function of $x$ for all values of $\eta$; hence, using Leibnitz' rule, and differentiating with respect to $x$, we obtain:

$$
\begin{aligned}
\frac{\partial \alpha_{\mu}}{\partial x}=\frac{1}{k_{\mu}^{2}} \int_{y+c}^{l} d_{\eta} \int_{x+c}^{l} u(\xi, \eta) D_{x}\left[1-(\xi-x)^{2}\right]^{\mu}\left[1-(\eta-y)^{2}\right]^{\mu} d \xi \\
-\frac{1}{k_{\mu}^{2}} \int_{v+\epsilon}^{1} u\left(x+\epsilon_{,} \eta\right)\left[1-\epsilon^{2}\right]^{\mu}\left[1-(\eta-y)^{2}\right]^{\mu} d \eta .
\end{aligned}
$$

Since the integrand of the second term is an absolutely continuous function of $x$ for all values of $\eta$ we can differentiate again, using Leibnitz' rule for Lebesgue integrals* and obtain:

$$
\begin{aligned}
& \frac{\partial^{2} \alpha_{\mu}}{\partial x^{2}}=\frac{1}{k_{\mu}^{2}} \int_{y+e}^{l} d \eta \int_{x+e}^{1} u(\xi, \eta) D_{z}^{2}\left[1-(\xi-x)^{2}\right]^{\mu}\left[1-(\eta-y)^{2}\right]^{\mu} d \eta \\
& -\frac{1}{k_{\mu}^{2}} \int_{v+\varepsilon}^{1} u(x+\epsilon, \eta)\left\{D_{x}\left[1-(\xi-x)^{2}\right]^{\mu}\right\}_{\xi=x+c}\left[1-(\eta-y)^{2}\right]^{\mu} d \eta \\
& -\frac{1}{k_{n}^{2}} \int_{v+\varepsilon}^{1} \frac{\partial}{\partial x} u(x+\epsilon, \eta)\left[1-\epsilon^{2}\right]+\left[1-(\eta-y)^{2}\right]^{\wedge} d \eta \text {. }
\end{aligned}
$$

- Cours d'analyse, Volume II, p. 123. 
A GREEN'S THEOREY IN TERMS OF LEBESGUE INTBGRALS. 147

The expressions for $\partial \alpha_{w} / \partial y$ and $\partial^{2} \alpha_{w} / \partial y^{2}$ are similar to those for $\partial \alpha_{w} / \partial x$, $\partial^{2} \alpha_{\mu} / \partial x^{2}$.

Differentiating $\beta_{n}[u(x, y)]$ we obtain:

$$
\begin{aligned}
& \frac{\partial \beta_{\mu}}{\partial x}=\frac{1}{k_{\mu}{ }^{2}} \int_{y-\varepsilon}^{y+\epsilon} d \eta \int_{x+c}^{l} u(\xi, \eta) D_{x}\left[1-(\xi-x)^{2}\right]^{\kappa}\left[1-(\eta-y)^{2}\right]^{\mu} d \xi \\
& -\frac{1}{k_{*}^{2}} \int_{y-\varepsilon}^{\nu+\varepsilon} u(x+\epsilon, \eta)\left[1-\epsilon^{2}\right]^{*}\left[1-(\eta-y)^{2}\right]^{\mu} d \eta \\
& \frac{\partial^{2} \beta_{\mu}}{\partial x^{2}}=\frac{1}{k_{*}^{2}} \int_{y-\varepsilon}^{++e} d \eta \int_{x+e}^{1} u(\xi, \eta) D_{x}^{2}\left[1-(\xi-x)^{2}\right]^{\mu}\left[1-(\eta-y)^{2}\right]^{\mu} d \xi \\
& -\frac{1}{k_{\mu}^{2}} \int_{y-\varepsilon}^{\mu+\bullet} u(x+\epsilon, \eta)\left\{D_{x}\left[1-(\xi-x)^{2}\right]\right\}_{\xi=x+\varepsilon[1}\left[1-(\eta-y)^{2}\right] \approx d \eta \\
& -\frac{1}{k_{u}^{2}} \int_{y-\varepsilon}^{\nu+e} \frac{\partial}{\partial x} u(x+\epsilon, \eta)\left[1-\epsilon^{2}\right]^{\mu}\left[1-(\eta-y)^{2}\right]^{\mu} d \eta \text {. }
\end{aligned}
$$

Writing $\beta_{k}$ in the form

$$
\frac{1}{k_{\mu}^{2}} \int_{x+c}^{1} d \xi \int_{y-\varepsilon}^{\nu+c} u(\xi, \eta)\left[1-(\xi-x)^{2}\right]^{\mu}\left[1-(\eta-y)^{2}\right]^{\mu} d \eta
$$

we obtain:

$$
\begin{aligned}
& \frac{\partial \beta_{\mu}}{\partial y}=\frac{1}{k_{*}^{2}} \int_{x+e}^{1} d \xi \int_{y-e}^{y+e} u(\xi, \eta)\left[1-(\xi-x)^{2}\right]^{\mu} D_{y}\left[1-(\eta-y)^{2}\right]^{*} d \eta \\
& +\frac{1}{k_{\mu}{ }^{2}} \int_{x+e}^{1} u(\xi, y+\epsilon)\left[1-(\xi-x)^{2}\right]^{\mu}\left[1-\epsilon^{2}\right]^{\mu} d \xi \\
& -\frac{1}{k_{\mu}{ }^{2}} \int_{x+\varepsilon}^{i} u(\xi, y-\epsilon)\left[1-(\xi-x)^{2}\right]^{\mu}\left[1-\epsilon^{2}\right]^{k} d \xi \text {, } \\
& \frac{\partial^{2} \beta_{\mu}}{\partial y^{2}}=\frac{1}{k_{k}^{2}} \int_{x+\varepsilon}^{l} d \xi \int_{y-\varepsilon}^{\psi+e} u(\xi, \eta)\left[1-(\xi-x)^{2}\right]^{\mu} D_{y}^{2}\left[1-(\eta-y)^{2}\right]^{\mu} d \eta \\
& +\frac{1}{k_{*}^{2}} \int_{x-\varepsilon}^{l} u(\xi, y+\epsilon)\left[1-(\xi-x)^{2}\right]^{*}\left\{D_{y}\left[1-(\eta-y)^{2}\right]^{\mu}\right\}_{\eta=r+e} d \xi \\
& -\frac{1}{k_{\mu}^{2}} \int_{x+e}^{1} u(\xi, y-\epsilon)\left[1-(\xi-x)^{2}\right]^{\mu}\left\{D_{y}\left[1-(\eta-y)^{2}\right]^{\mu}\right\}_{\eta=y+e} d \xi \\
& +\frac{1}{k_{\mu}^{2}} \int_{x+\varepsilon}^{1} \frac{\partial}{\partial y} u(\xi, y+\epsilon)\left[1-(\xi-x)^{2}\right]^{\kappa}\left[1-\epsilon^{2}\right]^{\mu} d \xi \\
& -\frac{1}{k_{k}^{2}} \int_{x+c}^{1} \frac{\partial}{\partial y} u(\xi, y-\epsilon)\left[1-(\xi-x)^{2}\right]^{\mu}\left[1-\epsilon^{2}\right]^{\mu} d \xi \text {. }
\end{aligned}
$$

V. From the expressions for $\partial \alpha_{\mu} / \partial x, \partial \alpha_{\mu} / \partial y, \partial \beta_{\mu} / \partial x, \partial \beta_{k} / \partial y$ we can now deduce the fact that $(\partial / \partial x) Q_{\alpha}[u]$ is an absolutely continuous function 
of $x$, and that $(\partial / \partial y) Q_{k}[u]$ is absolutely continuous in $y$. For, consider $\partial \alpha_{\mu} / \partial x$. The first term is of the same character as $\alpha_{\mu}$ itself, which is the sum of terms of the type

$$
A x^{i} y^{j} \int_{x+e}^{l} d \xi \int_{y+e}^{l} \xi \eta^{e} u(\xi, \eta) d \eta
$$

which is obviously, absolutely continuous since $u$ is limited. The second term satisfies the conditions of Theorem $B$, and is therefore an absolutely continuous function of $x$, for all values of $y$. The same kind of reasoning can be applied to the discussion of $\partial \alpha_{\mu} / \partial y, \partial \alpha_{\mu} / \partial x, \partial \beta_{\mu} / \partial y$ and we can infer therefore that $(\partial / \partial x) Q_{\mu}[u],(\partial / \partial y) Q_{\mu}[u]$ are absolutely continuous functions, respectively, of $x$ and $y$.

VI. We have next to show that $\left(\partial^{2} / \partial x^{2}\right) Q_{\mu}[u]$ and $\left(\partial^{2} / \partial y^{2}\right) Q_{\mu}[u]$ are summable, superficially, in the rectangle $S$. It will be seen that the only terms in the expressions representing $\partial^{2} \alpha_{\mu} / \partial x^{2}, \partial^{2} \alpha_{\mu} / \partial y^{2}, \partial^{2} \beta_{\mu} / \partial x^{2}, \partial^{2} \beta_{\mu} / \partial y^{2}$ whose summability is not evident are of a form of which the following is typical:

$$
\frac{1-\epsilon^{2}}{k_{\mu}^{2}} \int_{x+\varepsilon}^{1} \frac{\partial}{\partial y} u(\xi, y-\epsilon)\left[1-(\xi-x)^{2}\right]^{\mu} d \xi .
$$

The presence of the factor $\left[1-(\xi-x)^{2}\right]^{\mu}$ in the integrand will evidently not affect the discussion. We shall therefore consider the function

$$
F(x, y)=\int_{x+c}^{1} \frac{\partial}{\partial y} u(\xi, y-\epsilon) d \xi=\frac{\partial}{\partial y} \int_{x+c}^{1} u(\xi, y-\epsilon) d \xi
$$

and change the variable of integration by putting

$$
\xi=x+\epsilon+(1-x-\epsilon) t \text {. }
$$

We thus obtain

$$
F(x, y)=\int_{0}^{1} \frac{\partial}{\partial y} u[x+\epsilon+(1-x-\epsilon) t, y-\epsilon][1-x-\epsilon] d t .
$$

Consider now the function $(\partial / \partial Y) u(X, Y)$ which by hypothesis, is summable in the square $[0 \leq X \leq 1,0 \leq Y \leq 1]$. If we define the function

$$
u(X, Y, T)=u(X, Y)
$$

for all values of $T$, it is evident that $(\partial / \partial Y) u[X, Y, T]$ is summable in the three-dimensional region $[0 \leq X \leq 1,0 \leq Y \leq 1,0 \leq T \leq 1]$. It is therefore summable in any measurable subregion of this cube, for instance in the region $E(X, Y, T)$ bounded by the planes

$$
T=a, T=b ; Y=c-\epsilon, Y=d-\epsilon ; X=T+\epsilon, X=1 .
$$


A GREEN's THBOREM IN TERMS OF LEBESGUE INTEGRALS. 149

Let us now perform the following transformation: using the results of Theorem $A$

$$
\begin{array}{lrl}
X=x+\epsilon+(1-\epsilon-x) l, & J=\left|\begin{array}{ccc}
1-\ell & 0 & 1-\epsilon-x \\
0 & 1 & 0 \\
1 & 0 & 0
\end{array}\right| \\
T=x, \epsilon, & |J|=1-\epsilon-x .
\end{array}
$$

$(\partial / \partial Y) u[X, Y, T]=(\partial / \partial Y) u[X, Y]$ thus becomes

and the integral,

$$
\frac{\partial}{\partial Y} u[x+\epsilon+(1-\epsilon-x) t, y-\epsilon]
$$

$$
\begin{aligned}
\int_{E(X, Y, T)} & \iint \frac{\partial}{\partial y} u[X Y T] d X d Y d T \\
= & \int_{t=0}^{l} \int_{y=c}^{d} \int_{x=a}^{b} \frac{\partial}{\partial y} u[x+\epsilon+(1-\epsilon-x) t, y-\epsilon][1-\epsilon-x] d x d y d t .
\end{aligned}
$$

We have now shown that $(\partial / \partial y) u[x+\epsilon+(1-\epsilon-x) t, y-\epsilon][1-\epsilon-x]$ is summable in the region corresponding to $E(X, Y, T)$, i.e., in $[a \leq x \leq b$, $c \leq y \leq d, 0 \leq t \leq 1]$. We therefore know that $F(x, y)$ is summable in $S,{ }^{*}$ and we can write, in fact

$$
\begin{aligned}
\iint F(x, y) d x d y \\
\quad=\int_{S} \int d x d y \int_{0}^{1} \frac{\partial}{\partial y} u[x+\epsilon+(1-\epsilon-x) t, y-\epsilon][1-\epsilon-x] d t .
\end{aligned}
$$

VII. We next show that the absolute continuity of

$$
\int_{s} \int \Delta^{2} Q_{\mu} d x d y
$$

is uniform with regard to $\mu$. The term

$$
\frac{\left(1-\epsilon^{2}\right)^{\mu}}{k_{\mu}{ }^{2}} \int_{x+e}^{1} \frac{\partial}{\partial y} u(\xi, y-\epsilon)\left[1-(\xi-x)^{2}\right]^{\mu} d \xi
$$

is typical of those which go to make up $\Delta^{2} Q_{\mu}[u]$. Ignoring the factor $\left(1-\epsilon^{2}\right)^{\mu} / k_{\mu}^{2}$ which approaches zero as $\mu \doteq \infty$, and writing

$$
\begin{aligned}
& \iiint \pi_{\mu}(x, y, t) d t d x d y \\
&=\int_{c}^{d} \int_{a}^{b} \int_{0}^{t} \frac{\partial}{\partial y} u[x+\epsilon+(1-\epsilon-x) t, y-\epsilon][1-\epsilon-x] \\
& \times\left[1-\{\epsilon+(1-\epsilon-x) t\}^{2}\right]^{\mu} d t d x d y
\end{aligned}
$$

- Cours d'analyse, volume II, pp. 117 et seq. 
we see that

$$
\pi_{k}(x, y, t)=h(x, y, t)\left[1-\{\epsilon+(1-\epsilon-x) t\}^{2}\right]^{k}
$$

and that $\left|x_{k}(x, y, t)\right| \leq|h(x, y, t)|$, where

$$
h(x, y, t)=(\partial / \partial y) u[x+\epsilon+(1-\epsilon-x) t, y-\epsilon][1-\epsilon-x] \text {. }
$$

We have shown that $h(x, y, t)$ is summable in the region $S^{\prime}[a \leq x \leq b$, $c \leq y \leq d, 0 \leq t \leq 1]$. Hence $|h(x, y, t)|$ is summable in $S^{\prime}$ and $\pi_{\mu}$ the product of two summable functions, one of which is limited, is also summable in $S^{\prime}$, therefore

$$
\int_{S} \int d x d y \int_{0}^{1} \pi_{\mu}(x, y, t) d t \leq \int_{s} \int d x d y \int_{0}^{t}|h(x, y, t)| d t .
$$

Since $h$ is independent of $\mu$ we see from this equation that the absolute continuity of the integrals

$$
\iint d x d y \int_{0}^{1} \pi_{v}[x, y, t] d t
$$

is uniform is $S$, and our statement is proved.

VIII. We need to show that $(\partial / \partial x) Q_{k}[u]$ and $(\partial / \partial y) Q_{\mu}[u]$ are continuous functions of the two variables $x$ and $y$. It will be sufficient to consider the quantity $\partial \alpha_{\mu} / \partial x$.

The first term of $\partial \alpha_{\mu} / \partial x$ is like $\alpha_{\mu}$ itself, which is a sum of terms of the type

$$
A x^{i} y^{j} \int_{\eta+e}^{1} d \eta \int_{x+e}^{1} \xi \eta^{e} u(\xi, \eta) d \xi
$$

which is continuous if the integral itself is continuous. But the integral has a limited integrand and is evidently a continuous function of $(x, y)$.

The second term of $\partial \alpha_{n} / \partial x$ is a sum of terms of the type

$$
B y^{i} \int_{y+e}^{t} \eta^{r} u(x+\epsilon, \eta) d \eta,
$$

which is a continuous function of $x$ and $y$ if the integral is itself continuous. But the integral is of the type considered in Theorem $D$, and is therefore continuous. We have now proved that:

$\partial Q_{k} / \partial x, \partial Q_{k} / \partial y$ are continuous functions of $x$ and $y$ in $S$,

$\partial Q_{\mu} / \partial x$ is an absolutely continuous function of $x$ in $S$,

$\partial Q_{\mu} / \partial y$ is an absolutely continuous function of $y$ in $S$.

$\nabla^{2} Q_{\mu}$ is summable in $S$.

In fact, if we remember that $c$ is less than $a, 1-b, c, 1-d$, it becomes evident that the above statements apply also to the rectangle $\bar{S}\left[a-\epsilon^{\prime} \leq x \leq b+\epsilon^{\prime}, c-\epsilon^{\prime} \leq y \leq d+\epsilon^{\prime}\right]$, where $\epsilon^{\prime}$ is a small positive 
constant such that

$$
\epsilon+\epsilon^{\prime}<a, 1-b, c, 1-d .
$$

We can now apply Green's Formula, and obtain for any square \& inside of $\bar{S}$, such that 8 contains the point $\left(x_{0}, y_{0}\right)$.'

$$
\int_{0} \frac{\partial Q_{\mu}}{\partial x} d y-\frac{\partial Q_{\mu}}{\partial y} d x=\int_{s} \int \nabla^{2} Q_{\mu} d x d y
$$

Therefore, since $\nabla^{2} Q_{\mu} d x d y$ is summable,

$$
\lim _{m(s)=0} \frac{1}{m(8)} \int_{c} \frac{\partial Q_{\mu}}{\partial x} d y-\frac{\partial Q_{\mu}}{\partial y} d x=\nabla^{2} Q_{n}\left[u\left(x_{0}, y_{0}\right)\right]
$$

for nearly every point $\left(x_{0}, y_{0}\right)$ of $\bar{S}$, consequently of $S$.

IX. Consider now the expression

$$
\frac{\partial}{\partial x} \bar{P}_{\mu}[u(x, y)]=\frac{1}{k_{\mu}^{2}} \int_{-\varepsilon}^{e} d \eta \int_{-\varepsilon}^{e} \frac{\partial}{\partial x} u(\xi+x, \eta+y)\left[1-\xi^{2}\right]^{*}\left[1-\eta^{2}\right]^{*} d \xi \text {. }
$$

It is easy to show by means of Theorem $A$, as we applied it to the function $(\partial / \partial y) u[x+\epsilon+(1-\epsilon-x) t, y-\epsilon]$, that the function

$$
\frac{\partial}{\partial x} u(\xi+x, \eta+y)
$$

is summable in the three-dimensional region

$$
[-\epsilon \leq \xi \leq \epsilon, a \leq x \leq b,-\epsilon \leq \eta \leq \epsilon] \text {. }
$$

Hence we can write, treating $\partial u / \partial y$ in the same way,

$\frac{1}{\sigma} \int_{c} \frac{\partial}{\partial x} \bar{P}_{\mu}[u] d y-\frac{\partial}{\partial y} \bar{P}_{\mu[u] d x}$

$$
\begin{aligned}
& =\frac{1}{k_{\kappa}^{2}} \int_{-e}^{\varepsilon} d \eta \int_{-c}^{c}\left[1-\xi^{2}\right]^{\mu}\left[1-\eta^{2}\right]^{\mu} \frac{1}{\sigma} \int_{c}^{c} \frac{\partial u(\xi+x, \eta+y)}{\partial x} d y \\
& -\frac{\partial u(\xi+x, \eta+y)}{\partial y} d x \\
& =\frac{1}{k_{\kappa}^{2}} \int_{-e}^{e} d \eta \int_{-e}^{e}\left[1-\xi^{2}\right]^{*}\left[1-\eta^{2}\right]^{*} \frac{1}{\sigma} \int_{-} \int f(\xi+x, \eta+y) d x d y,
\end{aligned}
$$

where $\sigma$ is the area of a square containing the point $\left(\xi+x_{0}, \eta+y_{0}\right)$ as center. Let us choose a sequence of numbers $\sigma_{i}$ approaching zero as a limit as $\mu \neq \infty$. Then, if the absolute continuity of the integrals

$$
\int_{-i}^{e} d \xi \int_{-i}^{e} d \eta\left(1-\xi^{2}\right)^{\mu}\left(1-\eta^{2}\right)^{\mu} \frac{1}{\sigma_{i}} \int_{-i} \int f(\xi+x, \eta+y) d x d y
$$

\footnotetext{
* Cours d'analyze, II, p. 124.
} 
is uniform with regard to $i$ over the rectangle $[-\epsilon \leq \xi \leq \epsilon,-\epsilon \leq \eta \leq \epsilon]$, we can write

$$
\begin{aligned}
\lim _{\epsilon=0} \frac{1}{\sigma} \int \frac{\partial}{\partial x} \bar{P}_{\mu}[u] d y- & \frac{\partial}{\partial y} \bar{P}_{\mu}[u] d x \\
& =\frac{1}{k_{\mu}^{2}} \int_{-e}^{e} d \eta \int_{-e}^{\mu}\left(1-\xi^{2}\right)^{\mu}\left(1-\eta^{2}\right)^{\mu} f\left(x_{0}+\xi, y_{0}+\eta\right) d \xi .
\end{aligned}
$$

To prove that such is the case we can ignore the factor $\left[1-\xi^{2}\right]^{\mu}\left[1-\eta^{2}\right]^{\mu}$, which is positive and limited, and consider the integral

$$
\begin{aligned}
\int_{-a}^{\bullet} d \xi \int_{-i}^{e} d \eta \frac{1}{\sigma_{i}} \int_{-,} \int f(\xi+x, & +y) d x d y \\
& =\int_{R(e, \odot)} \iiint \frac{1}{\sigma_{i}} f(\xi+x, \eta+y) d \xi d \eta d x d y,
\end{aligned}
$$

since, as is easily shown by Theorem $A, f(\xi+x, \eta+y)$ is summable in the four-dimensional region

$$
\begin{aligned}
R[\epsilon, \sigma] \text { or }[-\epsilon \leq \xi \leq \epsilon,-\epsilon \leq \eta \leq \epsilon, \\
\left.\qquad x_{0}-\frac{\sqrt{\sigma_{i}}}{2} \leq x \leq x_{0}+\frac{\sqrt{\sigma_{i}}}{2}, y_{0}-\frac{\sqrt{\sigma_{i}}}{2} \leq y \leq y_{0}+\frac{\sqrt{\sigma_{i}}}{2}\right] .
\end{aligned}
$$

The last integral can be written

$$
\int_{x_{0}-\frac{\sqrt{\sigma_{i}}}{2}}^{\sigma_{0}+\frac{\sqrt{\sigma_{i}}}{2}} d x \int_{y_{0}-\frac{\sqrt{\sigma_{i}}}{2}}^{y_{0}+\frac{\sqrt{\sigma_{i}}}{\sigma_{i}}} d y \frac{1}{\sigma_{i}} \int_{-i}^{e} \int_{-i}^{e} f(\xi+x, \eta+y) d \xi d \eta
$$

Now, since $f$ is summable two-dimensionally in the fundamental region $R(0 \leq X \leq 1,0 \leq Y \leq 1)$, by the definition of absolute continuity of an integral,

$$
\mid \int_{e(\xi, \eta)} \int f(\xi+x, \eta+y) d \xi d \eta
$$

approaches zero with the measure of $e(\xi, \eta)$ (which is a part of the rectangle $(-\epsilon \leq \xi \leq \epsilon,-\epsilon \leq \eta \leq \epsilon)$ independent of $x$ and $y$, provided only that $(x, y)$ lie in the rectangle $S$. Hence

$$
\begin{aligned}
\iint_{y_{0}-\frac{\sqrt{\sigma_{i}}}{2}}^{v_{0}+\frac{\sqrt{\sigma_{i}}}{2}} d y \int_{x_{0}-\frac{\sqrt{\sigma_{i}}}{2}}^{x_{0}+\frac{\sqrt{\sigma_{i}}}{2}} d x \frac{1}{\sigma_{i}} \int_{e(\xi, \eta)} \int f(\xi+x, \eta & +y) d \xi d \eta \\
& \leq \int_{y_{0}-\frac{\sqrt{\sigma_{\sigma_{i}}}}{2}}^{y_{0}+\frac{\sqrt{\sigma_{i}}}{2}} d y \int_{x_{0}-\frac{\sqrt{\sigma_{i}}}{2}}^{r_{0}+\frac{\sqrt{\sigma_{1}}}{2}} d x \cdot \frac{1}{\sigma_{i}} \cdot \omega \\
& \leq \omega,
\end{aligned}
$$


where $\omega$ approaches zero with the measure of $e(\xi, \eta)$ independently of $x, y$. Hence

$$
\begin{aligned}
\lim _{\sigma=0} \frac{1}{\sigma} \int_{c(\sigma)} \frac{\partial}{\partial x} \bar{P}_{\mu}[u] d y & -\frac{\partial}{\partial y} \bar{P}_{\mu}[u] d x \\
& =\frac{1}{k_{\mu}{ }^{2}} \int_{-e}^{e} d \eta \int_{-e}^{e}\left[1-\xi^{2}\right]^{\mu}\left[1-\eta^{2}\right]^{\mu} f\left(x_{0}+\xi, y_{0}+\eta\right) d \xi .
\end{aligned}
$$

But we have proved that

$$
\lim _{\sigma=0} \frac{1}{\sigma} \int_{c(\sigma)} \frac{\partial}{\partial x} \bar{Q}_{\mu}[u] d y-\frac{\partial}{\partial y} Q_{\mu}[u] d x=\nabla^{2} Q_{\mu}\left[u\left(x_{0}, y_{0}\right)\right]
$$

Hence by addition

$$
\begin{aligned}
\lim _{\sigma=0} \frac{1}{\sigma} \int_{e(e)} \frac{\partial}{\partial x} P_{\mu}[u] d y-\frac{\partial}{\partial y} P_{\mu}[u] d x \\
\quad=\nabla^{2} Q_{\mu}\left[u\left(x_{0} y_{0}\right)\right]+\frac{1}{k_{\mu}{ }^{2}} \int_{-\varepsilon}^{e} d \eta \int_{-\varepsilon}^{e}\left(1-\xi^{2}\right)^{\mu}\left(1-\eta^{2}\right)^{\mu} f\left(x_{0}+\xi, y_{0}+\eta\right) d \xi .
\end{aligned}
$$

Therefore, since $f$ is summable and $\lim _{\mu=\infty} \nabla^{2} Q_{\mu}\left[u\left(x_{0}, y_{0}\right)\right]=0$,

$$
\lim _{\mu=\infty} \nabla^{2} P_{\mu}\left[u\left(x_{0}, y_{0}\right)\right]=f\left(x_{0}, y_{0}\right)
$$

at nearly every point $\left(x_{0}, y_{0}\right)$ of the region $S$.

Similarly we can write

$$
\lim _{\mu=\infty} \nabla^{2} P_{\mu}\left[v\left(x_{0}, y_{0}\right)\right]=g\left(x_{0}, y_{0}\right) \text {. }
$$

$X$. We now have to discuss the absolute continuity of the integrals appearing in equation (1), with regard to uniformity.

The functions $P_{\mu}[u], P_{\mu}[v]$ are limited in their sets: they will therefore not affect the discussion. We therefore consider the integral

$$
\begin{aligned}
\int_{c}\left[\frac{\partial}{\partial x} P[u(x, y)]\right]_{x=a} d y=\int_{c}^{d}\left[\frac{\partial}{\partial x} \bar{P}_{\mu}[u(x, y)]\right]_{x=a} d y \\
+\int_{c}^{d}\left[\frac{\partial}{\partial x} Q_{\mu}[u(x, y)]\right]_{x=a} d y,
\end{aligned}
$$

for which we have to prove the uniform absolute continuity.

I say that the quantities $\partial \alpha_{\mu} / \partial x, \partial \beta_{\mu} / \partial x$ which are representative of those which make up $\partial Q_{\mu} / \partial x$ are limited in their sets. For the integrands in the expression for $\partial \alpha_{\mu} / \partial x$ both contain the factor $\left[1-(\eta-y)^{2}\right]^{\mu}$ $\bar{\Sigma}\left(1-\epsilon^{2}\right)^{\mu}$ and since $\left(1 / k_{\mu}{ }^{2}\right)\left(1-\epsilon^{5}\right)^{\mu}$ approaches zero as $\mu \pm \infty, u$ being limited, it is evident that the functions $\left[\partial \alpha_{\mu} / \partial x\right]$ are limited in their 
set. Moreover, the integrands in the expression for $\partial \beta_{\mu} / \partial x$ contain the factors $D_{x}\left[1-(\xi-x)^{2}\right]^{k}$ and $\left(1-\epsilon^{2}\right)^{*}$ respectively. But since $D_{s}\left[1-(\xi-x)^{2}\right]^{k}$ is an infinitesimal of the order of $\mu\left[1-(\xi-x)^{2}\right]^{\alpha-1}$ $<\mu\left(1-\epsilon^{2}\right)^{\kappa-1}$ and since

$$
\frac{\mu\left(1-\epsilon^{2}\right)^{\mu-1}}{k_{\mu}^{2}} \div 0
$$

as $\mu=\infty$, we see that the functions $\left[\partial \beta_{\mu} / \partial x\right]$ are limited in their set. It follows therefore that the functions $\left[\partial Q_{E} / \partial x\right]$ are limited in their set, and obviously approach zero everywhere in $D$.

Hence

and

$$
\lim _{x=\infty} \int_{c}^{d}\left[\frac{\partial Q_{x}[u(x, y)]}{\partial x}\right]_{x=a} d y=0
$$

$$
\lim _{\mu=\infty} \int_{c}^{d}\left[\frac{\partial}{\partial x} P_{\mu}[u(x, y)]\right]_{x=a} d y=\lim _{\mu=\infty} \int_{e}^{d}\left[\frac{\partial}{\partial x} \bar{P}_{\mu}[u(x, y)]\right]_{x=\mathrm{a}} d y \text {. }
$$

We have now to show that the absolute continuity of the integral

$$
\int_{a}^{\mu}\left[\frac{\partial}{\partial x} \bar{P}_{\mu}[u(x, y)]\right]_{x=a} d y
$$

is uniform with regard to $\mu$. Now

$$
\begin{aligned}
\int_{c}^{d}\left[\frac{\partial}{\partial x} \bar{P}_{\mu}[u(x, y)]\right]_{x=a} d y \\
\quad=\int_{c}^{\alpha} d y \frac{1}{k_{k}^{2}} \int_{-e}^{e} \int_{-e}^{e}\left[\frac{\partial}{\partial y} u(\xi+x, \eta+y)\right]_{x=a}\left(1-\xi^{2}\right)^{\mu}\left(1-\eta^{2}\right)^{\mu} d \xi d \eta .
\end{aligned}
$$

By means of Theorem $A$, it is easy to show, by the method of VI that $[(\partial / \partial x) u(\xi+x, \eta+y)]$ is summable in the three-dimensional region

$$
[-\epsilon \leq \xi \leq \epsilon,-\epsilon \leq \eta \leq \epsilon, c \leq y \leq d] \text {, }
$$

we can therefore write

$$
\begin{aligned}
\int_{c}^{d}\left[\frac{\partial}{\partial x}\right. & \left.\bar{P}_{\mu}[u(x, y)]\right]_{x=a} d y \\
\quad & \frac{1}{k_{\mu}^{2}} \int_{-c}^{e} d \xi \int_{-c}^{e} d \eta\left[1-\xi^{2}\right]^{\mu}\left[1-\eta^{2}\right]^{\mu} \int_{c}^{d}\left[\frac{\partial}{\partial x} u(\xi+x, \eta+y)\right]_{x=a} d y .
\end{aligned}
$$

We now make use of the statement (6) of our hypothesis, that the absolute continuity of the integral

$$
\int \frac{\partial}{\partial x} u(x, y) d y
$$


is uniform in the neighborhood of $x=a$. So far we have not made use of the fact that the constant $\epsilon$ can be chosen as small as we please. We now choose $\epsilon$ so small that the absolute continuity of $\mathcal{S}(\partial / \partial x) u(x, y) d y$ will be uniform for all values of $x$ in the interval $a-\epsilon \leq x \leq a+c$.

Then

$$
\left|\int_{c(s)}\left[\frac{\partial}{\partial x} \bar{P}_{\mu}[u(x, y)]\right]_{x=0} d y\right| \leq\left|\frac{1}{k_{\mu}^{2}} \int_{-e}^{e} d \xi \int_{-e}^{e} d \eta\left(1-\xi^{2}\right)^{\mu}\left(1-\eta^{2}\right)^{\mu} \omega\right| \text {, }
$$

where $\omega \neq 0$ with $m[e(y)]$ independent of $\xi$ and $\eta$. Thus our statement is proved.

XI. With regard to the right-hand member of equation (1) we proceed as follows:

We have shown that

$$
\nabla^{2} P_{\mu}[u]=\nabla^{2} Q_{\mu}[u]+\frac{1}{k_{\mu}^{2}} \int_{-e}^{e} d \eta \int_{-e}^{e}\left(1-\xi^{2}\right)^{\kappa}\left(1-\eta^{2}\right)^{\mu} f\left(x_{0}+\xi, y_{0}+\eta\right) d \xi
$$

and we have shown that the absolute continuity of $\int \mathcal{S} \nabla^{2} Q_{\mu}[u] d x d y$ is uniform in $S$. We have now to show that the absolute continuity of

$$
\begin{aligned}
\iint \bar{P}_{\mu}[f(x, y)] d x d y & \\
& =\frac{1}{k_{\mu}{ }^{2}} \iint d x d y \int_{-\varepsilon}^{e} \int_{-\varepsilon}^{e} f(x+\xi, y+\eta)\left(1-\xi^{2}\right)^{\mu}\left(1-\eta^{2}\right)^{\mu} d \xi d \eta
\end{aligned}
$$

is uniform in $S$.

Since, as we have stated before, $f(x+\xi, \eta+y)$ is summable in the four-dimensional region

$$
[a \leq x \leq b, c \leq y \leq d,-\epsilon \leq \xi \leq \epsilon,-\epsilon \leq \eta \leq \epsilon],
$$

we can write.

$$
\begin{aligned}
& \iint \bar{P}_{\mu}[f(x, y)] d x d y \\
& \quad=\frac{1}{k_{*}^{2}} \int_{-i}^{c} d \xi \int_{-i}^{e} d \eta\left(1-\xi^{2}\right)^{*}\left(1-\eta^{2}\right)^{*} \int_{s} \int f(x+\xi, y+\eta) d x d y .
\end{aligned}
$$

But since $f(x, y)$ is summable in $R[0 \leq x \leq 1,0 \leq y \leq 1]$ by the definition of absolute continuity, since $\epsilon<a, 1-b, c, 1-d$, the absolute continuity of

$$
\int \mathcal{S} f(x+\xi, y+\eta) d x d y
$$

is uniform for all values of the parameters $\xi, \eta$ in the region

$$
[-\epsilon \leq \xi \leq \epsilon,-\epsilon \leq \eta \leq \epsilon] \text {. }
$$


Hence

$$
\mid \int_{e(x, y)} \int \bar{P}_{\mu}[f(x, y)] d x d y \leq \frac{1}{k_{\mu}{ }^{2}} \int_{-\epsilon}^{{ }_{\epsilon}^{\epsilon}} d \xi \int_{-\epsilon}^{\epsilon} \omega\left[1-\xi^{2}\right]^{\mu}\left[1-\eta^{2}\right]^{\mu} d \eta \leq \omega,
$$

where $\omega \doteq 0$ with $m[e(x, y)]$ independent of $\xi$ and $\eta$. The proof is now complete; for in view of the nature of the rectangle $S$, we know that $(\partial / \partial x) P_{\mu}[u],(\partial / \partial y) P_{\mu}[u]$ approach $\partial u / \partial x, \partial u / \partial y$ nearly everywhere along its boundary.

Moreover $\nabla^{2} P_{\mu}[u]$ approaches $f(x, y)$ nearly everywhere in $D$. Similar statements apply to $(\partial / \partial x) P_{\mu}[v],(\partial / \partial y) P_{\mu}[v]$ and to $\nabla^{2} P_{\mu}[v]$. Taking the limits of both sides of equation (1) as $\mu \doteq \infty$ we obtain finally

$$
\int_{c}\left(v \frac{\partial u}{\partial x}-u \frac{\partial v}{\partial x}\right) d y-\left(v \frac{\partial u}{\partial y}-u \frac{\partial v}{\partial y}\right) d x=\int_{S} \int(v f-u g) d x d y \text {. }
$$

The Rice Institute. 